\title{
Clostridial (Blackleg) Diseases of Cattle 1
}

\section{E. J. Richey, DVM²}

Diseases including blackleg, malignant edema, sord, black disease, red water, and three kinds of enterotoxemia are all caused by a genus of bacteria called Clostridium. These bacteria have three important qualities: they multiply only in the absence of oxygen; they have the ability to survive adverse conditions by transforming into highly resistant forms called spores; and they release potent toxins during the process of multiplying. It is the combination of these three characteristics that make the clostridia highly dangerous.

In their spore form, clostridia are able to exist in the soil, on body surfaces and within the healthy animal. The clostridia do not cause disease until tissue is damaged to provide a favorable environment where the dormant clostridial spores are able to transform into active multiplying bacteria. During this multiplication, potent toxins are released that can destroy muscle tissue, destroy red blood cells, or interrupt nerve impulses, depending upon which clostridial organism is present. Basically, the principal infection sites of clostridial bacteria are the muscles, the liver and the intestines. Thus, the clostridial diseases are categorized into muscle group, liver group, and gastrointestinal (gut) group.

\section{Muscle Group of Clostridia}

These clostridial organisms usually enter the body through wounds and lie dormant until a suitable environment for their activation occurs. Any injury, that interferes with the blood circulation to tissues, will create an environment in which $\mathrm{Cl}$. chauvoei, $\mathrm{Cl}$. septicum and $\mathrm{Cl}$. sordellii can be activated. Bruises caused by butting, riding, restraint procedures, shipping, and close confinement around feed bunks; scratches; wounds; and damage to lung tissue from pneumonia are examples of the injuries that will often create a suitable environment. The toxins released by these multiplying bacteria produce widespread muscle damage and impair the functions of vital organs. These three clostridial species are referred to as the muscle-destroying clostridia and cause blackleg, malignant edema, and sord disease respectively. All three diseases are characterized by swelling, collecting of fluids in the tissues, and sometimes production of gas in the muscle tissues surrounding the area of infection. The swellings are seen most often in the area of the hip, shoulder, neck and upper leg. Affected animals are usually lame and depressed, have an elevated body temperature during the early phases of the disease, and exhibit a drop in the body temperature to below normal as the disease

1. This document is VM48, one of a series of the Veterinary Medicine-Large Animal Clinical Sciences Department, Florida Cooperative Extension Service, Institute of Food and Agricultural Sciences, University of Florida. Original publication date June 1, 1987. Reviewed and revised April, 1996, December, 2004 Visit the EDIS Web Site at http://edis.ifas.ufl.edu.

2. E.J. Richey, Extension Veterinarian, College of Veterinary Medicine, Cooperative Extension Service, Institute of Food and Agricultural Sciences, University of Florida, Gainesville, 32611.

The Institute of Food and Agricultural Sciences (IFAS) is an Equal Employment Opportunity - Affirmative Action Employer authorized to provide research, educational information and other services only to individuals and institutions that function without regard to race, creed, color, religion, age, disability, sex, sexual orientation, marital status, national origin, political opinions or affiliations. For information on obtaining other extension publications, contact your county Cooperative Extension Service office. Florida Cooperative Extension Service / Institute of Food and Agricultural Sciences / University of Florida / Larry R. Arrington, Interim Dean 
progresses. In the final stages, the depression worsens and the animal goes down. Death usually occurs within 12 hours after the appearance of clinical disease. Because of the rapid death, most cattle producers find the affected animals dead, rather than sick.

\section{Liver Group of Clostridia}

The spores of the liver group enter the body through contaminated feed and water, become established in the intestinal tract, and eventually enter the bloodstream for distribution to the liver. Some form of liver damage is required for the activation of the spores. Common causes of liver damage include liver abscess, chemical or plant poisoning, fatty changes in the liver, internal parasites, and liver flukes. The Liver Group consists of two clostridia, $\mathrm{Cl}$. novyi and $\mathrm{Cl}$. haemolyticum. In the presence of liver damage, the dormant spores become activated and multiply. As with the muscle group, potent toxins are produced while the bacteria are multiplying. The toxins expand the area of liver damage and are absorbed into the bloodstream, through which they reach and damage vital organs.

The diseases of the liver group are black disease caused by Cl.novyi and bacillary hemoglobinuria (red water) caused by $\mathrm{Cl}$. haemolyticum. These diseases follow a rapid course and the affected animals are often found dead rather than sick. Early signs include depression, fever, tendency to stand apart from the herd, and reluctance to move. Many affected animals assume an arched back posture with the neck extended. As the disease progresses, breathing becomes difficult and blood-tinged froth may appear in the nostrils. In lingering cases of $\mathrm{Cl}$.

haemolyticum, the urine may turn red in color, hence the name red water or bacillary hemoglobinuria.

\section{Gastrointestinal (Gut) Group of Clostridia}

The gastrointestinal (gut) group of clostridia consists of three types of $\mathrm{Cl}$. perfringens which cause enterotoxemia disease. As a group $\mathrm{Cl}$. perfringens have been found in the intestinal tracts of most animals and are worldwide in distribution. After entering the body in feed and water, the $\mathrm{Cl}$. perfringens revert from the spore form to the non-spore form in the small intestine. Conditions that reduce oxygen availability can stimulate multiplication of the bacteria and their release of potent toxins. The diseases of the gastrointestinal group appear following the intake of feeds high in soluble carbohydrates and/or when the diet is changed suddenly. Excess ingestion of feed brings about a change in the rumen organisms with a subsequent increase in lactic acid, producing acidosis. In addition, partially fermented grain is allowed to enter the small intestines mainly in the form of starch granules. This creates an environment favoring rapid multiplication of Type D Cl. perfringens. As acidosis worsens, the motility of the rumen and the intestinal tract decreases. This allows the toxin produced by the multiplying Type $\mathrm{D}$ bacteria to accumulate in the forward part of the small intestine where it eventually escapes into the blood stream. Type $\mathrm{C} \mathrm{Cl}$. perfringens is activated under conditions in the small intestines produced by large quantities of milk, reduced intestinal motility, and even lack of exercise. Upon activation, the Type $\mathrm{C}$ bacteria multiply and produce large quantities of potent toxins that damage the gut and are absorbed into the bloodstream. Type B Cl. perfringens has not been regarded as a significant problem in the United States, even though some cases have been diagnosed.

Deaths from diseases of the gut group occur suddenly, usually before clinical signs are seen. Type $\mathrm{D}$ disease symptoms include excitement or convulsions. These signs are caused by the nerve tissue effects of the toxin produced by the multiplying bacteria. In Type $\mathrm{C}$ disease, signs include abdominal pain or colic, depression, and "low bloat." Type C symptoms are a result of the tissue-destroying toxin that produces severe inflammation and hemorrhage of the intestinal lining, often referred to as "purple gut."

\section{Protecting Your Cattle}

The need for pre-challenge vaccination to raise the resistance level is of critical importance in controlling all clostridial diseases because, first, clostridial spores reside within the animal's body and are widely distributed in the soil; second, the presence of spores in the animal does not stimulate resistance to the activated form of the organism or to 
the toxins it releases; and third, the activated clostridia cause death before the animal can mount an adequate defense response.

With the exception of $C l$. chauvoei (blackleg), two doses of vaccine are required to stimulate resistance against all clostridial bacteria because a high level of antibodies must be immediately available to combat the active bacteria and/or their toxins. The first dose of the vaccine stimulates the production of a small amount of short-acting antibodies and the second dose stimulates a secondary response which results in a large amount of long-lasting antibodies. Animals being vaccinated against any of the clostridial diseases for the first time should receive two doses three to four weeks apart. Animals vaccinated under three months of age should be revaccinated at weaning or at four to six months of age.

Clostridial vaccines have been shown to cause injection site blemishes in beef; therefore, it is recommended that clostridial vaccines, when labeled as such, be administered under the skin (subcutaneously) of the neck or behind the shoulder.

To maximize the resistance of brood cows and to help assure that their calves receive high levels of resistance from the first milk, all animals should be booster vaccinated at least once a year. To provide adequate resistance against the liver group $(\mathrm{Cl}$. novyi $\&$ Cl. haemolyticum ), all animals should be booster vaccinated every six months. A guiding rule for the timing of booster vaccinations should be to booster vaccinate before periods of high risk. Calves are at high risk when they are nursing and taking in a lot of rich mother's milk; when they are growing on creep feed or milk replacers; when they are being castrated, dehorned, or shipped; when they are growing on lush pastures or suffering from respiratory diseases; and when they are finishing in the feed yard. Brood cows and bulls are at high risk when they are grazing pastures infested with snails (the intermediate host of the liver fluke), grazing lush pastures, and supplementing with a concentrated feed.

Cattle that die from clostridial infections should be completely burned or covered with quicklime and deeply buried. The clostridium organism is distributed throughout the carcass and will be a source of infection to other cattle or be transmitted by predators, scavengers or rain water to other premises.

It has been shown that properly vaccinating calves with a bacterin containing the muscle, liver and gut clostridia (8-way) reduced the death loss in a western feedyard by $47.3 \%$. The significant reduction in death loss was not surprising; a feedyard is an ideal site for concentrating the clostridium organisms that are transported by the cattle from various geographical regions and is considered a high-risk time in the calves' lives.

Blackleg organisms are just waiting for an accident to happen; then they cause disease. This is why we should vaccinate calves for clostridial (blackleg) diseases before shipment. 